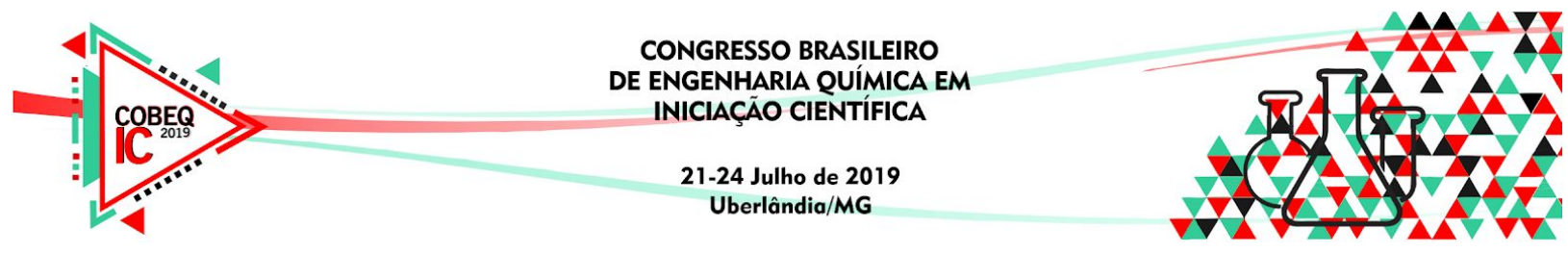

\title{
DESENVOLVIMENTO DE UM SISTEMA DE CONTROLE INTELIGENTE EM UM PROTÓTIPO EXPERIMENTAL DE FLOTAÇÃO POR AR DISSOLVIDO PARA TRATAMENTO DE ÁGUA
}

\author{
V. ROZANTE ${ }^{1}$, A. C. O. SOUZA ${ }^{1}$, F. V. DA SILVA ${ }^{1}$ \\ ${ }^{1}$ Universidade Estadual de Campinas, Faculdade de Engenharia Química, Departamento de \\ Engenharia de Sistemas Químicos, \\ E-mail para contato: flavio@feq.unicamp.br
}

RESUMO - Os processos químicos são, em geral, não lineares e inerentemente multivariáveis, exibindo fortes interações entre as variáveis de processos, muitas vezes sujeitas a restrições. Com o avanço da tecnologia, os sistemas e processos químicos ficaram mais complexos, tornando-se uma tarefa árdua a utilização satisfatória dos controladores convencionais obtidos a partir da teoria clássica de controle. Analisando este panorama, propôs-se o desenvolvimento de um sistema e automação aplicado a um protótipo experimental de flotação por ar dissolvido (FAD) que permitiu a implementação de uma estratégia de controle inteligente baseado em lógica fuzzy. A flotação por ar dissolvido é um processo amplamente utilizado em tratamento de água e efluentes. Nela, as partículas em suspensão têm sua densidade aparente reduzida a partir da adesão de microbolhas de ar, o que provoca a separação de tais partículas. Devido à natureza multivariável, os processos de tratamento de água são difíceis de controlar e justificam a utilização de uma abordagem baseada em controladores avançados inteligentes. $O$ controlador fuzzy desenvolvido conseguiu atingir satisfatoriamente os valores de turbidez definidos em diferentes valores de referência (set point).

\section{INTRODUÇÃO}

Um tema recorrente quando se discute sociedade e tecnologia é em relação ao uso da água e ao saneamento básico, necessidade das cidades para que as populações tenham uma boa qualidade de vida. Diante disso, as estações de tratamento de água (ETAs) e as estações de tratamento de efluentes (ETEs) são essenciais para garantir a distribuição de uma água adequada para consumo humano e para promover o tratamento dos efluentes gerados nos processos industriais.

\subsection{Sistema de Flotação por Ar Dissolvido (FAD)}

Dentre as técnicas empregadas durante as etapas de clarificação do tratamento de água para abastecimento público tem-se a flotação por ar dissolvido (FAD). A aplicação do sistema 


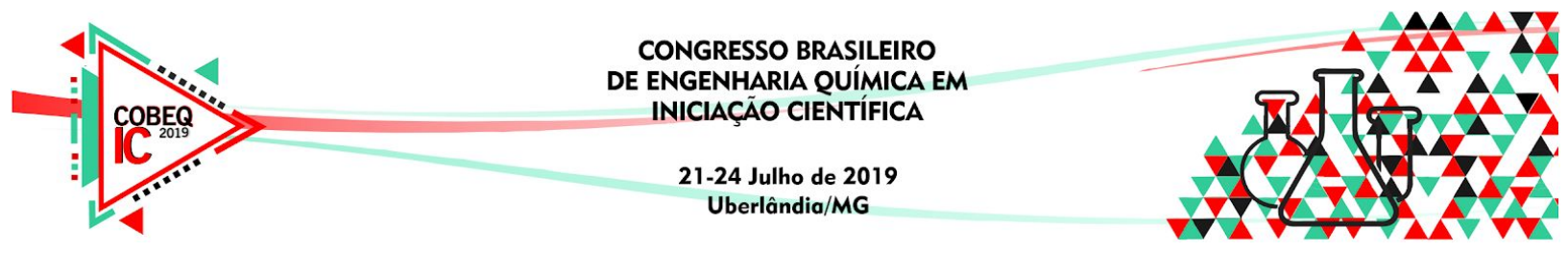

de FAD voltada ao tratamento de água começou a ser vastamente utilizada nos países escandinavos no período entre 1960 e 1970. O crescente interesse na utilização deste método deve-se ao fato das vantagens que o mesmo apresenta, quanto à remoção dos sólidos em suspensão quando comparado aos processos tradicionalmente utilizados nas ETAs, como a sedimentação (KIURI, 2001).

\subsection{Lógica Fuzzy}

Tradicionalmente os computadores utilizam-se da lógica clássica, mais precisamente a lógica booleana, na qual os conjuntos são separados e bem definidos, aos que pertencem e não pertencem a uma classe. É notável que tal situação, na maioria dos casos, não reflete fielmente aos problemas da realidade a serem modelados. Tendo isto em mente, a Lógica Fuzzy (ou Lógica Difusa), representa a maneira com que o ser humano pensa e possui como finalidade simular o senso comum e tomadas de decisão.

A estrutura do processo Fuzzy é iniciado com uma etapa de fuzzyficação, seguida de um processo de defuzzyficação e, por fim, é a determinação de uma ação de controle não-fuzzy que represente fielmente a decisão tomada, podendo ser enviada ao controle proposto. O método de defuzzificação mais comum, e usado neste trabalho, é o Centro de Gravidade. Neste a obtenção do sinal de saída do controlador ocorre no centro de massa da área de intersecção entre as áreas obtidas pela projeção dos valores das variáveis de entrada em suas funções de pertinência (FONSECA, 2017).

Tendo isto em vista, a não linearidade deste tipo de controlador está atrelado a geometria com que as MF's serão manipuladas, que podem variar entre sigmoidal, trapezoidal e triangular. Como apresentado por FONSECA (2017) e GHANSEM (2006), a conformação mais usual para o procedimento de controle Fuzzy é a partir do formato triangular, pois estas são mais fáceis de projetar a respostas do processo e obtendo bons resultados.

\section{MATERIAIS E MÉTODOS}

\subsection{Protótipo de Flotação por Ar Dissolvido e Ensaios Realizados}

Fonseca (2017) realizou a construção, controle e instrumentação do protótipo de flotação por ar dissolvido, representado na Figura 1. A linha de pesquisa desenvolvida no Laboratório de Controle e Automação de Processos (LCAP) da Faculdade de Engenharia Química da UNICAMP estuda a aplicação da FAD ao tratamento de água para consumo. Portanto, para a realização dos ensaios no protótipo de FAD preparou-se uma suspensão sintética a partir de terra vermelha (saibro), para simular as características da água bruta que é captada em rios e enviada às ETAs do Brasil.

Após o preparo do efluente sintético, soluções de Tanino 5\% v/v e $\mathrm{NaAlO}_{2} 2 \% \mathrm{v} / \mathrm{v}$ foram utilizadas nas etapas de coagulação e floculação. A dosagem destas substâncias químicas foi específica para cada valor de turbidez inicial da água bruta e foi estabelecida por 


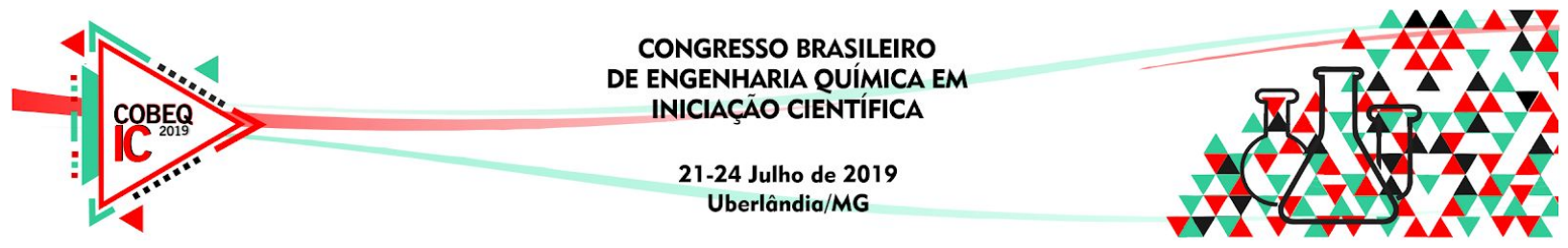

ensaios de teste de jarro (jar tests). Por ensaios preliminares, determinaram-se as vazões ideais dos agentes floculantes e coagulantes do processo FAD para uma determinada turbidez. $\mathrm{Na}$ Tabela 1 é mostrada as condições de processos que foram adotadas nos ensaios de aplicação do controlador Fuzzy.

Figura 1 - Protótipo de flotação por ar dissolvido do LCAP/FEQ/UNICAMP.

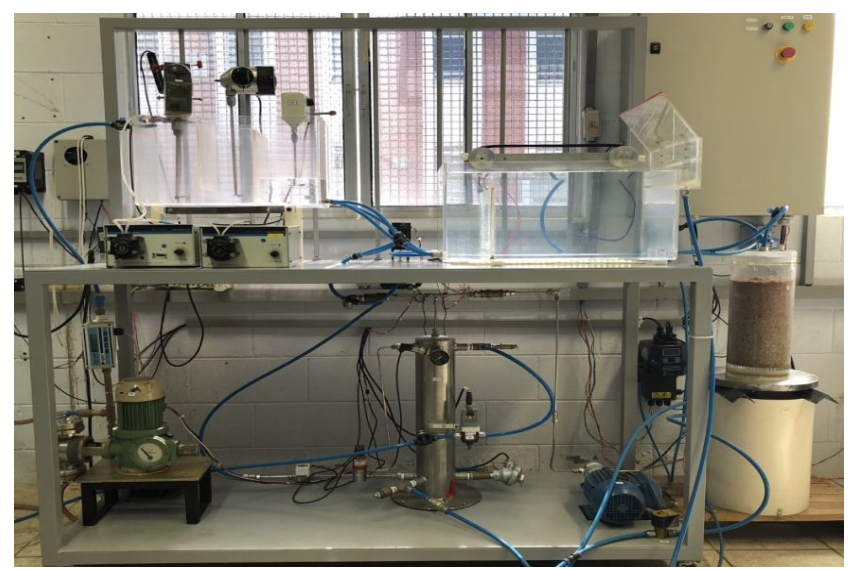

Fonte: SOUZA, 2019.

Tabela 1 - Condições experimentais utilizadas nos ensaios no protótipo de FAD.

\begin{tabular}{|c|c|c|c|c|}
\hline $\begin{array}{c}\text { Turbidez } \\
\text { inicial (NTU) }\end{array}$ & $\begin{array}{c}\text { Pressão } \\
\text { (bar) }\end{array}$ & $\begin{array}{c}\text { Vazão de floculante } \\
(\mathrm{mL} / \mathrm{min})\end{array}$ & $\begin{array}{c}\text { Vazão de coagulante } \\
(\mathrm{mL} / \mathrm{min})\end{array}$ & $\begin{array}{c}\text { Vazão de água } \\
\text { bruta (L/min) }\end{array}$ \\
\hline 28 & 6 & 8 & 2 & 2 \\
\hline
\end{tabular}

\subsection{Ensaios com o controlador Fuzzy}

De modo a se aplicar um controle inteligente ao processo de FAD estudado, utilizou-se um sistema baseado em múltiplas variáveis de entrada com apenas uma variável de saída, denominada como a variável manipulada. Esta estratégia é denominada como MISO e suas variáveis de entrada são o erro da turbidez ( $e_{T U}$ ) e a derivada do erro $\left(d e_{T U}\right)$. A escolha da derivada do erro como uma variável de entrada serviu como um norte para analisar o comportamento dinâmico da turbidez ao longo do processo experimental, assim como demonstrado por Fonseca (2017).

A estratégia de controle utilizada foi baseada na atuação de uma variável manipulada (MV) a partir da medição dinâmica de uma variável do processo, denominada como variável controlada (PV). A variável controlada neste trabalho foi a turbidez final, medida por um 


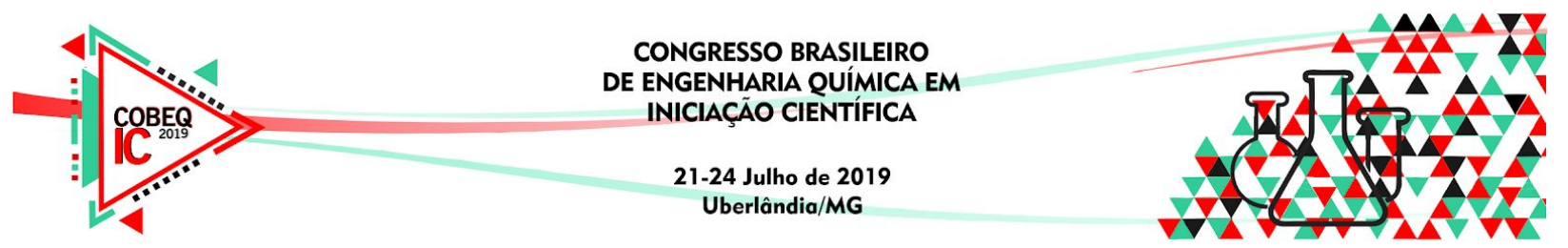

turbidímetro online posicionado na saída do tanque de flotação, e a variável manipulada foi a porcentagem de abertura da válvula agulha.

A automação e controle da planta de Flotação por Ar Dissolvido foi realizada com o auxílio da ferramenta Fuzzy Controller, inclusa no pacote Simulink/MATLAB®, no qual foi aplicado o controle da planta em malha aberta e malha fechada de subsistemas, tais como, a vazão de efluente, posição da válvula agulha, vazão de reciclo, dentre outros.

\section{RESULTADOS E DISCUSSÃO}

O controlador fuzzy foi construído com um universo de discurso normalizado em NTU para ambas variáveis de entrada (erro da turbidez e derivada do erro), assim como para a variável de saída. De maneira análoga, para as mesmas variáveis, foram propostas 5 funções de pertinência baseadas nos seguintes termos linguísticos: Muito Baixo (MB), Baixo (B), Zero (Z), Alto (A) e Muito Alto (MA). Desta forma, foram criadas para este controlador fuzzy 25 regras, baseadas em conhecimento especialista, como apresentado na Tabela 2 . A variável de saída representa o incremento na variável manipulada, em \%, o qual foi configurada com 5 funções de pertinência utilizando os mesmos termos linguísticos das variáveis de entrada. As regras propostas foram criadas da seguinte forma:

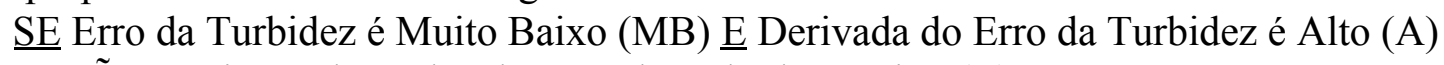

ENTÃO Variação do \% de Abertura da Válvula é Baixo (B).

Tabela 2 - Base de regras para o controlador Fuzzy projetado.

\begin{tabular}{|c|c|c|c|c|c|}
\hline$d e_{T U} / e_{T U}$ & $\mathrm{MB}$ & $\mathrm{B}$ & $\mathrm{Z}$ & $\mathrm{A}$ & $\mathrm{MA}$ \\
\hline $\mathrm{MB}$ & $\mathrm{MB}$ & $\mathrm{MB}$ & $\mathrm{MB}$ & $\mathrm{B}$ & $\mathrm{Z}$ \\
\hline $\mathrm{B}$ & $\mathrm{MB}$ & $\mathrm{MB}$ & $\mathrm{B}$ & $\mathrm{Z}$ & $\mathrm{A}$ \\
\hline $\mathrm{Z}$ & $\mathrm{MB}$ & $\mathrm{B}$ & $\mathrm{Z}$ & $\mathrm{A}$ & $\mathrm{MA}$ \\
\hline $\mathrm{A}$ & $\mathrm{B}$ & $\mathrm{Z}$ & $\mathrm{A}$ & $\mathrm{MA}$ & MA \\
\hline $\mathrm{MA}$ & $\mathrm{Z}$ & $\mathrm{A}$ & MA & MA & MA \\
\hline
\end{tabular}

A superfície de controle apresentada na Figura 2 representa a atuação do controlador configurado, Pode-se observar que esta é mais intensa em situações nas quais $e_{T U} e d e_{T U}$ estão mais próximos de seus limites de universo de discurso proposto e, também, é observável um comportamento suave na atuação da variável de entrada com relação a de saída. A variável do erro do controlador está no espaço $[-1,1]$, enquanto que derivada do mesmo está em $[-1,1]$ e a integral do erro está no espaço $[-1,1]$.

Na Figura 3 é apresentada a malha de controle de turbidez da água pós-flotação utilizando o controlador Fuzzy. Neste sistema, a pressão interna do vaso saturador foi mantida 


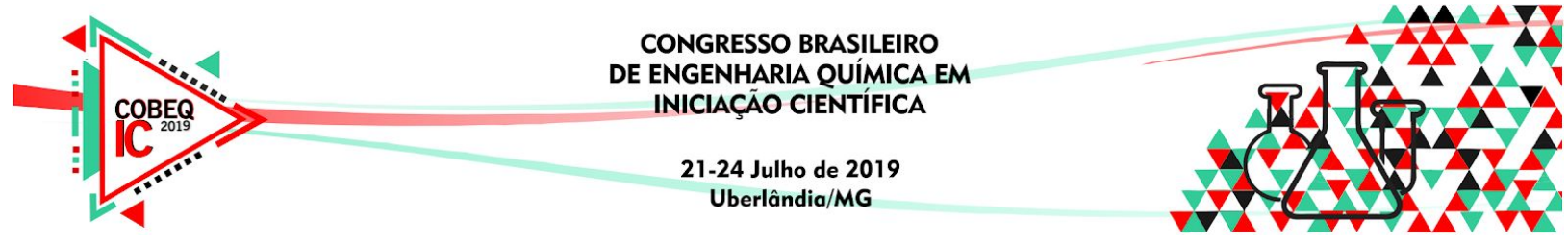

em um valor constante igual a 6 bar, valor dentro da faixa permitida do equipamento, sendo a variável manipulada a abertura da válvula agulha.

Na Figura 4(a) é mostrado o comportamento dinâmico da variável controlada (turbidez de saída) na estratégia de controle adotada. Inicialmente o processo foi mantido em regime permanente com a turbidez de saída em 28 NTU e, posteriormente, foram realizadas perturbações servo nos valores de setpoint da turbidez (20 NTU, 10 NTU, 4 NTU).

Figura 2 - Superfície resposta para o controlador Fuzzy proposto.

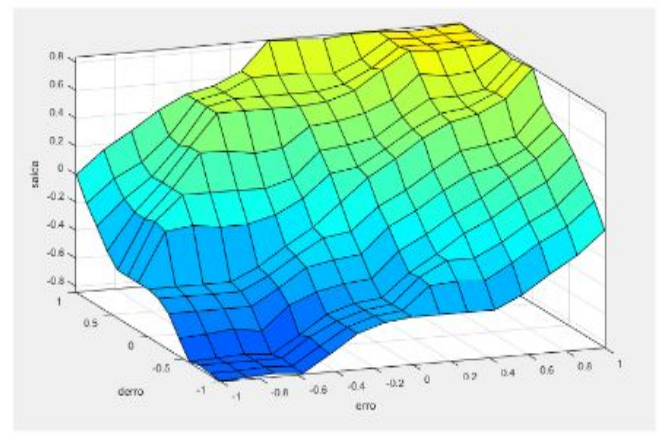

Figura 3 - Malha de controle da turbidez pós-flotador utilizando lógica Fuzzy.

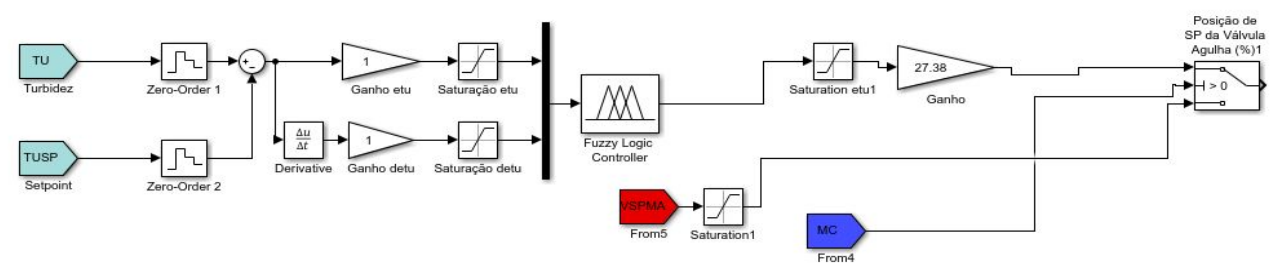

Figura 4 - Comportamento dinâmico da turbidez de saída durante o ensaio com o controlador Fuzzy (a) Perfil temporal da abertura da válvula V01 durante o tempo de ensaio (b).

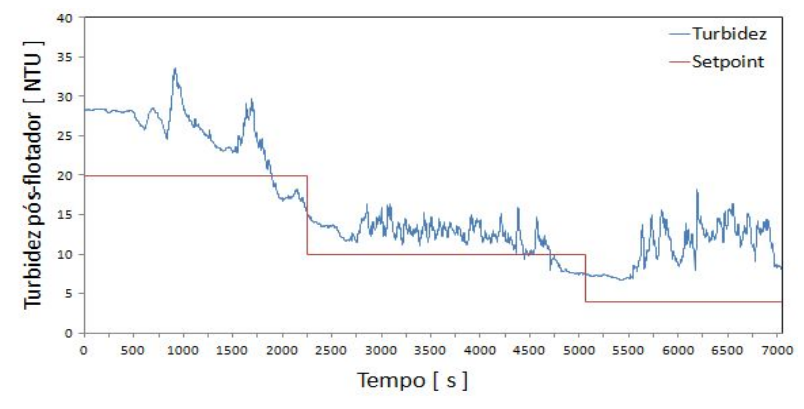

(a)

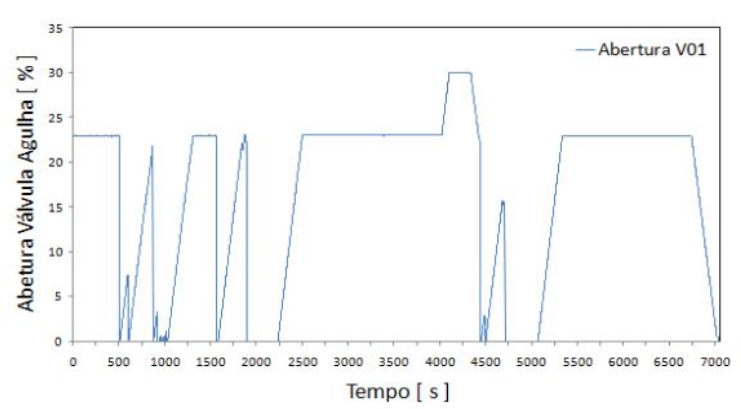

(b) 


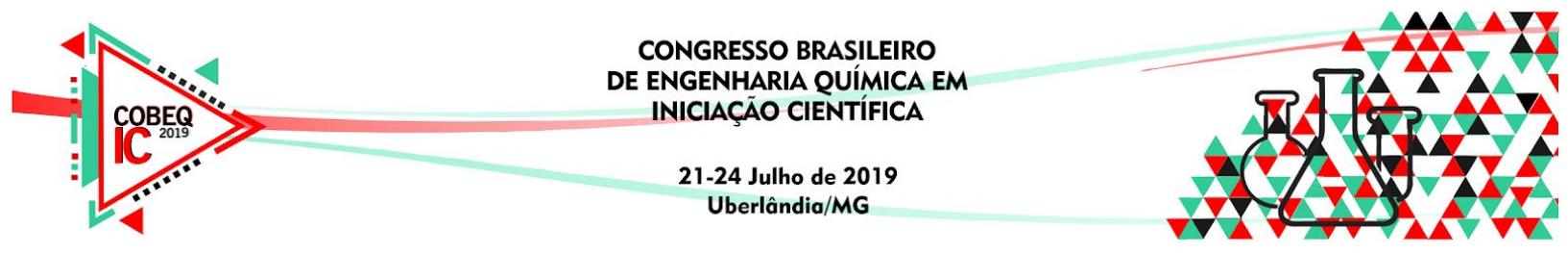

Analisando a atuação do controlador Fuzzy implementado, foi possível observar que o mesmo conseguiu atingir seu objetivo em reduzir a turbidez, entretanto de maneira não efetiva. O regime permanente da variável controlada manteve-se acima da condição de setpoint definida para os três casos. O caráter oscilatório do sinal de turbidez monitorado deve-se à posição da tomada de medição do turbidímetro online, que acaba captando pequenos flocos que decantaram, o que ocasionou os picos intermitentes na dinâmica temporal dessa variável. Para a Figura 4(b), com relação a abertura da válvula, foi observado que este parâmetro apresentou um caráter linear, não demonstrando um esforço muito grande no equipamento para atingir seu valor de setpoint. Observa-se claramente a necessidade de se realizar uma sintonia mais efetiva no controlador fuzzy implementado, principalmente nos ganhos de saída (atuação). Observa-se uma atuação agressiva que se traduz em um baixo desempenho do controlador fuzzy.

\section{CONCLUSÃO}

A implementação experimental de controladores Fuzzy utilizou-se da mesma automação disponível para os controladores convencionais, no qual o controle da turbidez da água efluente foi regulada atuando-se sobre a abertura da válvula agulha o qual mostrou-se prática, entretanto não apresentando bons resultados na regulação da variável manipulada.

É importante ressaltar que o sucesso da implementação dos controladores Fuzzy está diretamente relacionado ao conhecimento especialista do projetista do mesmo. Assim, em ensaios futuros será focado na alteração da base de regras e o universo de discurso das variáveis de entrada e saída do controlador Fuzzy para torná-lo menos sensível às perturbações e mais exato quanto o alcance do setpoint de turbidez adotado, além da implementação de degraus positivos para o controlador.

\section{REFERÊNCIAS}

ANG, K. H.; CHONG, G; LI, Y. PID control system analysis, design, and technology. IEEE transactions on control systems technology, v. 13, n. 4, p. 559-576, 2005.

BAHADORI, A. et al. Estimation of air concentration in dissolved air flotation (DAF) systems using a simple predictive tool. Chemical Engineering Research and Design, v. 91, n. 1, p. 184-190, 2013.

FONSECA, R. R. Monitoramento e controle avançado aplicados à flotação por ar dissolvido, 2017. Tese (Doutorado em Engenharia Química) - Faculdade de Engenharia Química, Universidade Estadual de Campinas.

GHANSEM, N. M. Design of fuzzy logic controller for regulating the temperature in industrial polyethylene fluidized bed reactor. Chemical Engineering Research and Design, v. 84(A2), p. 97 - 106, 2006. 


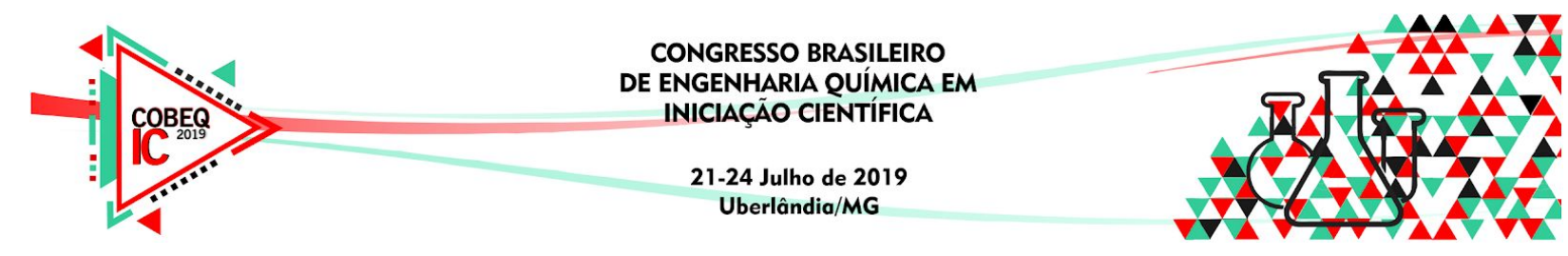

SOUZA, A. C. O. Modelagem empírica do comportamento dinâmico de um protótipo de flotação por ar dissolvido baseada em redes neurais artificiais, 2019. Dissertação (Mestrado em Engenharia Química) - Faculdade de Engenharia Química, Universidade Estadual de Campinas. 